\title{
Mevalonate Kinase Deficiency and Squalene Synthase Inhibitor (TAK-475): The Balance to Extinguish the Inflammation
}

\author{
Erika Rimondi ${ }^{1,2}$, Erica Valencic ${ }^{3}\left(\mathbb{D}\right.$, Alberto Tommasini ${ }^{3,4, *(D)}$, Paola Secchiero ${ }^{1,2}$, Elisabetta Melloni ${ }^{1,2,+(\mathbb{C})}$ \\ and Annalisa Marcuzzi ${ }^{1,+}$ (iD \\ 1 Department of Translational Medicine, University of Ferrara, 44121 Ferrara, Italy; \\ erika.rimondi@unife.it (E.R.); paola.secchiero@unife.it (P.S.); elisabetta.melloni@unife.it (E.M.); \\ annalisa.marcuzzi@unife.it (A.M.) \\ 2 LTTA Centre, University of Ferrara, 44121 Ferrara, Italy \\ 3 Department of Paediatrics, Institute for Maternal and Child Health-IRCCS “Burlo Garofolo", 34137 Trieste, \\ Italy; erica.valencic@burlo.trieste.it \\ 4 Department of Medical Sciences, University of Trieste, 34127 Trieste, Italy \\ * Correspondence: alberto.tommasini@burlo.trieste.it \\ + These authors equally contributed to this work.
}

check for updates

Citation: Rimondi, E.; Valencic, E.; Tommasini, A.; Secchiero, P.; Melloni, E.; Marcuzzi, A. Mevalonate Kinase Deficiency and Squalene Synthase Inhibitor (TAK-475): The Balance to Extinguish the Inflammation. Biomolecules 2021, 11, 1438. https:// doi.org/10.3390/biom11101438

Academic Editors: Saida Ortolano, Jorge Julian Fernández Martín and Beatriz San-Millán-Tejado

Received: 29 August 2021

Accepted: 27 September 2021

Published: 30 September 2021

Publisher's Note: MDPI stays neutral with regard to jurisdictional claims in published maps and institutional affiliations.

Copyright: (c) 2021 by the authors. Licensee MDPI, Basel, Switzerland. This article is an open access article distributed under the terms and conditions of the Creative Commons Attribution (CC BY) license (https:// creativecommons.org/licenses/by/ $4.0 /)$.
Abstract: Mevalonate Kinase Deficiency (MKD) is a rare inborn disease belonging to the family of periodic fever syndromes. The MKD phenotype is characterized by systemic inflammation involving multiple organs, including the nervous system. Current anti-inflammatory approaches to MKD are only partially effective and do not act specifically on neural inflammation. According to the new emerging pharmacology trends, the repositioning of drugs from the indication for which they were originally intended to another one can make mechanistic-based medications easily available to treat rare diseases. According to this perspective, the squalene synthase inhibitor Lapaquistat (TAK-475), originally developed as a cholesterol-lowering drug, might find a new indication in MKD, by modulating the mevalonate cholesterol pathway, increasing the availability of anti-inflammatory isoprenoid intermediates. Using an in vitro model for MKD, we mimicked the blockade of the cholesterol pathway and evaluated the potential anti-inflammatory effect of Lapaquistat. The results obtained showed anti-inflammatory effects of Lapaquistat in association with a low blockade of the metabolic pathway, while this effect did not remain with a tighter blockade. On these bases, Lapaquistat could be configured as an effective treatment for MKD's mild forms, in which the residual enzymatic activity is only reduced and not almost completely absent as in the severe forms.

Keywords: mevalonate; inflammation; drug repositioning; rare disease

\section{Introduction}

Mevalonate Kinase Deficiency (MKD) is a rare metabolic and autoinflammatory disorder caused by the mutation of the $M V K$ gene (chromosome 12, q24). The mutation leads to a reduction in the mevalonate kinase enzyme activity, with a consequent decrease in a series of isoprenoid intermediate compounds and a mevalonate accumulation in plasma and urine in the acute phase [1] (Figure 1).

The clinical onset of the disease occurs in pediatric age but tends to last in life, presenting various degrees of severity, ranging from the less debilitating form (hyperimmunoglobulinemia D, HIDS, OMIM \# 260920) to the most severe form (mevalonic aciduria, MA, OMIM \# 610377). Both phenotypes are characterized by common symptoms that include periodic fever attacks associated with other inflammatory symptoms [2,3]. The common symptoms in HIDS are headache, splenomegaly, adenopathy, pharyngitis, abdominal and musculoskeletal pain. MA is also characterized, in addition to these symptoms, by significant psycho-motor and neurological involvement [4]. 


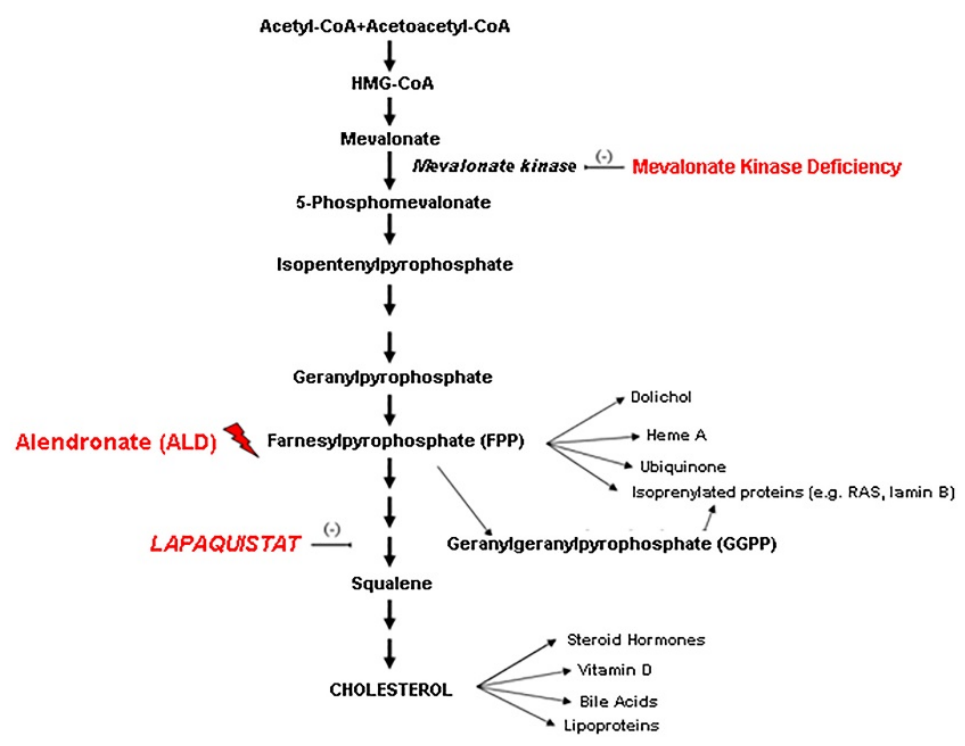

Figure 1. Schematic representation of the mevalonate pathway.

The most accredited hypothesis regarding MKD pathogenesis claims that the typical inflammation of the disease is caused by the deficiency of pre-squalenic isoprenoid intermediates, with a reduced prenylation of small GTPases, which, consequently, lose their membrane localization $[5,6]$. The lack of membrane-bound RhoA reflects on a reduced threshold of activation of the NLR family pyrin domain containing 3 (NLRP3) and pyrin inflammasomes and to the process of pyroptosis with a secretion of the inflammatory cytokines IL-1 $\beta$, IL-6 and TNF- $\alpha$ [7-9]. An altered prenylation of KRAS (an isoform of RAS) is thought to play a role in the activation of PI3K- $\delta$ and in the lymphoproliferative phenotype characterizing MKD. Furthermore, the incorrect prenylation of the small GTPase Rab11 does not allow the formation of the autophagosome, leading to impaired mitophagy, which may contribute to the neurological damage observed in the most serious forms of MKD [10]. To date, MKD is no longer considered a treatment-orphan disease, because the IL-1 inhibitor treatments have allowed an acceptable control of the disease in most cases. However, not all patients fully respond to these therapies [11]. Furthermore, the anti-IL-1 biological treatments have a very high cost and require parenteral administration [12]. It remains, therefore, advisable to deepen the knowledge about the pathogenesis of MKD to identify more specific molecular targets for a mechanistic treatment.

Recent in vitro studies have shown a possible therapeutic role of a biochemical modulation of the mevalonate pathway [13]. For example, Lapaquistat (TAK-475), a compound belonging to the family of zaragozic acid, has been reported as a powerful competitive inhibitor of cholesterol synthesis in HepG2 cells [14]. Similarly, in a murine model, zaragozic acid was able to inhibit acute liver cholesterol synthesis accompanied by an accumulation of organic acids [15]. In summary, these experimental data highlighted the potential of squalene inhibitors in hypercholesterolemia [16]. The effect of zaragozic acid is related not only to the blockade of sterol synthesis, but also to a relative accumulation of geranylgeraniol due to both the blocking of its conversion to squalene and the induction of the mevalonate kinase [17]. Preliminary studies also suggest that this action could also help to protect neurons from a toxic effect that occurs under isoprenoid insufficiency conditions [18].

Of note, zaragozic acid could improve the MK residual enzymatic activity in skin fibroblasts derived from patients with MKD, increasing the transcription of the gene Mvk [13].

According to all evidence, TAK- 475 could affect pathogenetic events relevant in MKD by increasing the levels of metabolites immediately upstream of the enzyme, including anti-inflammatory isoprenoids derived from the geraniol [19].

The study of the TAK- 475 is also justified from the availability of safety data of the compound in phase three clinical trials at a lower dosage (50 mg/daily). However, the drug 
at a higher concentration (100 mg/daily) was halted because of a hepatic safety concern in addition to its comparable effectiveness as a cholesterol-lowering agent with respect to statins $[20,21]$. Nevertheless, even at a lower dosage, TAK-475 treatment led to reduction in C-reactive protein, highlighting its anti-inflammatory potential [22].

Therefore, the aim of the present study was to evaluate the potential of TAK-475 in our MKD model and, at the same time, to improve the understanding of the pathogenetic mechanism correlated to MKD.

\section{Materials and Methods}

\subsection{Reagents}

Lipopolysaccharide (LPS) (E. coli-serotype 055:B5) and alendronate (ALD), (SigmaAldrich, St. Louis, MO, USA) were dissolved in saline solution. Lapaquistat acetate (TAK-475, Shanghai iChemical Technology, Shanghai, China; purity 98\%) was dissolved in ethanol before dilution in tissue culture medium, so that the final concentration of ethanol would not exceed $0.1 \%(\mathrm{vol} / \mathrm{vol})$.

\subsection{Cell Culture}

RAW 264.7 cells (murine monocyte/macrophage cell line), obtained from SigmaAldrich, were cultured in Dulbecco's modified eagle medium (DMEM) supplemented with $10 \%$ fetal bovine serum (both from EuroClone, Milan, Italy) and with L-glutamine and penicillin/streptomycin (GIBCO, Grand Island, NY, USA). Cells were left untreated or treated with 25 or $50 \mu \mathrm{M}$ ALD for $20 \mathrm{~h}$ and, then, with $10 \mu \mathrm{g} / \mathrm{mL}$ LPS for an additional $24 \mathrm{~h}$. TAK-475 (LAPA) was added together with ALD, when appropriate, at the concentration of $10 \mu \mathrm{M}$. At the end of the treatment time, the supernatants were collected for cytokines analyses, while cells were pelleted for the analysis of apoptosis, the electron microscopy studies and the Western blot assays.

\subsection{Flow Cytometric Assessment of Apoptosis}

The cytotoxic effects of ALD and LPS were assessed verifying apoptosis induction after Annexin V-FITC/propidium iodide (PI) double staining (Beckman Coulter Inc., Brea, CA, USA), according to the manufacturer's instructions, using a FACSCalibur flow cytometer (BD Biosciences, San Josè, CA, USA) and the FlowJo software, version 9.9.6 (Tree Star, Ashland, OR, USA). The percentage of apoptotic cells correspond to the sum of $\mathrm{A}+/ \mathrm{PI}+$ (late apoptotic) and A +/PI - (early apoptotic) cells as shown in the Supplementary Figure S1.

\subsection{The xCELLigence System and Impedance Measurement}

The effect of the ALD, LPS and LAPA treatment on RAW 264.7 was evaluated by the xCELLigence RTCA DP Instrument (F. Hoffmann-La Roche SA, Basel, Switzerland), which records the increase in electrical impedance due to the presence of adherent cells on the well bottom, covered by microelectrodes. In real time and without the addition of a label, the number and the viability of attached cells are displayed as an alteration of the impedance that is converted in an adimensional parameter called "Cell Index" (CI). A decrease in $\mathrm{CI}$ after adding a pharmacological compound could be due to a detachment or to the death of cultured cells. Briefly, for this analysis, $5 \times 10^{3}$ cells were seeded in a 16-well E-plate in $200 \mu \mathrm{L}$ of complete medium, and cultured in $5 \% \mathrm{CO}_{2}$ at $37^{\circ} \mathrm{C}$. The cells were stimulated with ALD, LPS and LAPA. The impedance was measured every $15 \mathrm{~min}$; this experiment was reproduced thrice in triplicate. The change of impedance was calculated by dividing the values by the value of the untreated condition.

\subsection{Measurement of Cytokines in Cell Culture Supernatants}

Cytokine levels were measured in RAW 264.7 cell culture supernatant samples by performing a bead-based multiplex immunoassay (Bio-Plex assay, Bio-Rad Laboratories, Milan, Italy), including IL-6 and TNF- $\alpha$, following the manufacturer's instructions. Data 
were acquired using the Bio-Plex 200 reader, while a digital processor managed data output and Bio-Plex Manager ${ }^{\circledR} 6.0$ software presented data as Median Fluorescence Intensity and concentration $(\mathrm{pg} / \mathrm{mL})$ as well (Bio-Rad Laboratories).

\subsection{Transmission Electron Microscopy}

For Transmission Electron Microscopy, cells were treated as described above. Cells were then collected and centrifuged at $2000 \times g$ for $5 \mathrm{~min}$ and the pellets were fixed in $2.5 \%$ glutaraldehyde in $0.1 \mathrm{M}$ phosphate-buffered saline (PBS, pH 7.4) at $4{ }^{\circ} \mathrm{C}$ for $3 \mathrm{~h}$. After several rinses with PBS, the cells were post-fixed in $2 \%$ buffered osmium tetroxide for $1 \mathrm{~h}$. The samples were then dehydrated with graded concentrations of acetone and embedded in Araldite epoxy resin (Durcupan ACM, Fluka, Sigma-Aldrich Co., St. Louis, MO, USA) according to standard protocols. Semi-thin sections $(1.5 \mu \mathrm{m})$ were cut on a Reichert Ultracut $S$ ultramicrotome using glass knives, stained with a $1 \%$ toluidine blue solution and observed with an optical microscope (Nikon Eclipse E800). Ultrathin sections $(90 \mathrm{~nm})$ were prepared with an ultramicrotome (Reichert Ultracut S) and counterstained with uranyl acetate in a saturated solution and lead citrate according to Reynolds. The samples were observed under transmission electron microscope (Hitachi H800 at $100 \mathrm{Kv}$, Hitachi, Ltd., Tokyo, Japan).

\subsection{Western Blot Analyses}

RAW 264.7 cells, cultured and treated as reported above, were lysed in ice-cold RIPA buffer ( $50 \mathrm{mM}$ Tris pH 7.5, $150 \mathrm{mM} \mathrm{NaCl}, 0.1 \%$ SDS, $1 \%$ Nonidet P- $40,0.25 \%$ sodium deoxycholate) supplemented with Pierce Protease and Phosphatase Inhibitor mini tablets (Thermo Scientific, Rockford, IL, USA) on ice for $1 \mathrm{~h}$. Protein determination was performed by using the BCA Protein Assay (Thermo Scientific, Rockford, IL, USA). Samples were supplemented with the loading buffer ( $250 \mathrm{mM}$ Tris pH 6.8, 2\% SDS, 40\% Glycerin, 20\% b-mercaptoethanol) and boiled for $2 \mathrm{~min}$. Equal amounts of protein for each sample were migrated in acrylamide gels and blotted onto nitrocellulose filters. Western blot analysis was performed according to standard procedures using the following primary antibodies: anti-LC3 and anti $\beta$-actin all from Sigma-Aldrich (St. Louis, MO, USA). After incubation with secondary antibodies (anti-mouse or -rabbit IgG HRP-conjugated; Sigma-Aldrich, St. Louis, MO, USA), a specific band detection was performed with the WesternBright Quantum kit (Advansta, Menlo Park, CA, USA). Images' acquisition and analysis were performed using the ImageQuant ${ }^{\mathrm{TM}}$ LAS 4000 imager and TL software (GE Healthcare, Buckinghamshire, UK).

\subsection{Data Analysis}

All results were expressed as mean \pm standard deviation. Statistical significance was calculated using two-way analysis of variance (ANOVA) and Bonferroni post-test. Analysis was performed through GraphPad Prism software, version 8.4.2 (GraphPad Software, La Jolla, CA, USA).

\section{Results}

\subsection{Alendronate Plus LPS Induced Apoptosis on RAW 264.7 Cells}

The effect on cell viability of the combination of alendronate (ALD) and lipopolysaccharide (LPS), that mimics the MKD disease condition, was evaluated on RAW 264.7 cells by flow cytometry after treatment with 25 or $50 \mu \mathrm{M}$ ALD for $48 \mathrm{~h}$, in addition to $10 \mu \mathrm{g} / \mathrm{mL}$ LPS for $24 \mathrm{~h}$. Results showed a significant induction of apoptosis after treatment with both 25 and $50 \mu \mathrm{M}$ ALD, confirming a potent cytotoxic effect of this drug combination in our cellular model (Figure 2) (Untreated: $11.24 \pm 4.24 \%$; ALD25 + LPS: $45.95 \pm 4.22 \%$; ALD50 + LPS: $56.45 \pm 0.83 \%$; **** $p<0.0001$ ). The single stimulus (ALD and LPS) did not induce a significant rate of apoptosis (ALD25: $21.38 \pm 7.22 \%$; ALD50: $24.50 \pm 9.80 \%$; LPS: $27.93 \pm 7.09 \%$ ) (data not shown). 


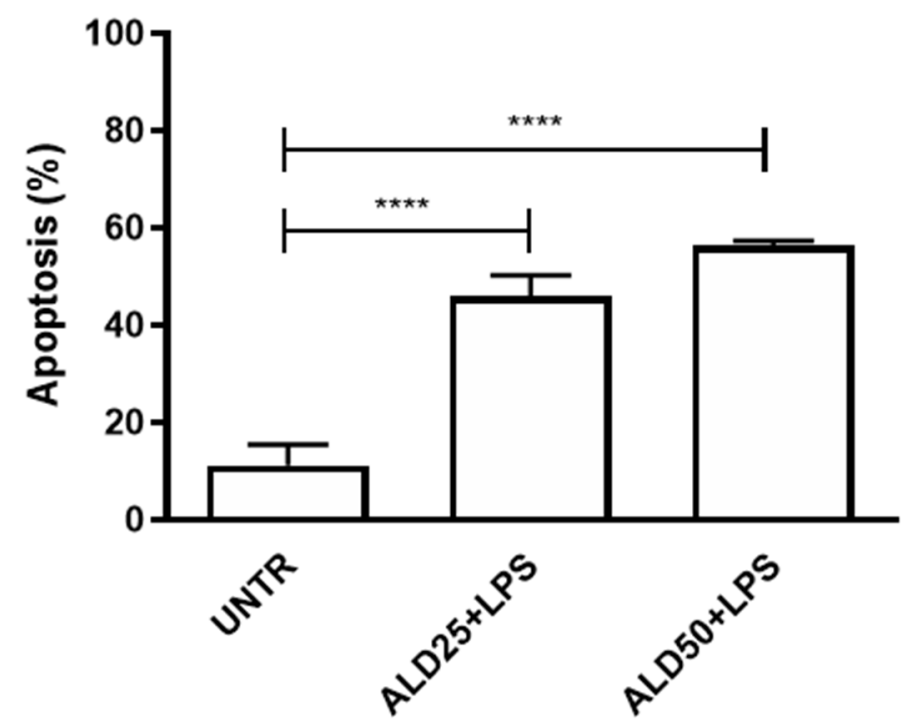

Figure 2. Analysis of RAW 264.7 apoptosis levels after treatment with two different ALD concentrations, $25 \mu \mathrm{M}$ and $50 \mu \mathrm{M})$ plus LPS $(10 \mu \mathrm{g} / \mathrm{mL})$. Results are indicated as mean \pm standard deviation of independent replicates, analyzed with ANOVA and corrected with Bonferroni post-test (**** $p<0.0001)$. UNTR: untreated.

\subsection{Lapaquistat Counteracted the Cytotoxic Effect of Alendronate Plus LPS}

The potential cytoprotective effect of Lapaquistat (LAPA) was evaluated on RAW 264.7 cells by the xCELLigence RTCA DP Instrument after $48 \mathrm{~h}$ of treatment with $10 \mu \mathrm{M}$ of the drug in combination with $25 \mu \mathrm{M}$ or $50 \mu \mathrm{M}$ ALD and $24 \mathrm{~h}$ of treatment with $10 \mu \mathrm{g} / \mathrm{mL}$ LPS.

As shown in Figure 3, treatment with ALD (at the highest concentration, $50 \mu \mathrm{M}$ ) plus LPS significantly reduced the Cell Index with respect to untreated cells and this effect was clearly counteracted by Lapaquistat, that partially restored the Cell Index value (Untreated: $100.1 \pm 21.33 \%$; ALD + LPS: $28.86 \pm 12.52 \%$; LAPA + ALD + LPS: $65.50 \pm 6.75 \%)$. The trend of ALD $25 \mu \mathrm{M}$ was perfectly comparable to that of ALD $50 \mu \mathrm{M}$ (data not shown). Of note, treatment with Lapaquistat alone did not show any cytotoxic effect in RAW 264.7 cells (LAPA: $108 \pm 19.18 \%$ ).

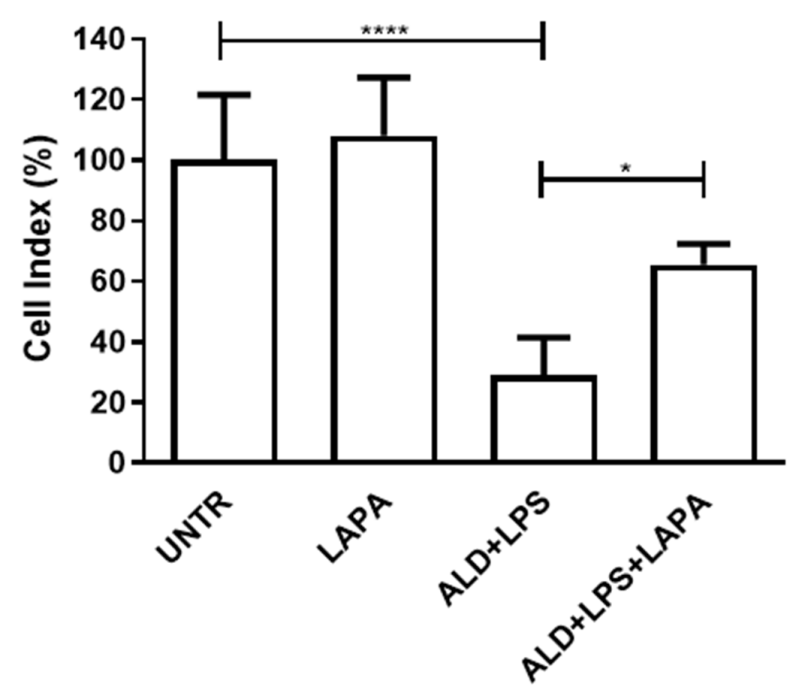

Figure 3. XCELLigence analysis of RAW 264.7 cell viability after treatment with ALD (50 $\mu$ M), LPS $(10 \mu \mathrm{g} / \mathrm{mL})$ and $10 \mu \mathrm{M}$ Lapaquistat (LAPA). Results are indicated as mean \pm standard deviation of 3 independent replicates, analyzed with ANOVA and corrected with Bonferroni post-test $\left({ }^{*} p<0.05\right.$, **** $p<0.0001)$. UNTR: untreated. 


\subsection{Anti-Inflammatory Effect of Lapaquistat in MKD Disease-Mimicking Conditions}

The levels of a wide panel of pro-inflammatory cytokines were firstly evaluated on RAW 264.7 cells treated for $48 \mathrm{~h}$ with 25 or $50 \mu \mathrm{M}$ ALD and for the last $24 \mathrm{~h}$ with $10 \mu \mathrm{g} / \mathrm{mL}$ LPS. Among the cytokines analyzed, the treatment induced a significant increase in IL-6 (Figure 4) and TNF- $\alpha$ levels (Figure 5), but did not seem to affect the release of the other cytokines in the culture supernatants (data not shown). The simultaneous addition of Lapaquistat to ALD plus LPS treatment significantly reduced the induced levels of IL-6 and TNF- $\alpha$ (IL-6. Untreated: $23.72 \pm 0.19$; ALD25 + LPS: $48.39 \pm 4.21$; ALD25 + LPS + LAPA: $28.26 \pm 6.66$; ALD50 + LPS: $84.07 \pm 2.03$; ALD50 + LPS + LAPA: $65.44 \pm 6.29)($ TNF- $\alpha$. Untreated: $23.43 \pm$ 10.57; ALD25 + LPS: $140.6 \pm 20.69$; ALD25 + LPS + LAPA: $49.41 \pm 8.92$; ALD50 + LPS: $404.7 \pm 78.19$; ALD50 + LPS + LAPA: $212.9 \pm 8.10)$. These data seemed to demonstrate the anti-inflammatory effect of Lapaquistat in our experimental MKD disease-mimicking setting.

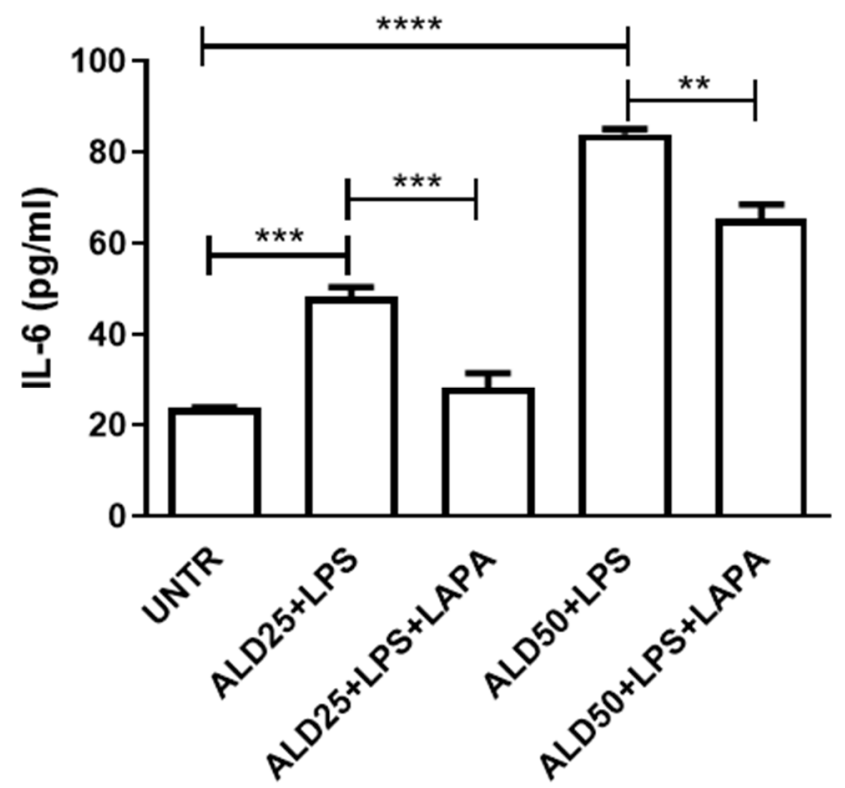

Figure 4. Analysis of IL-6 levels on supernatants of RAW 264.7 treated with ALD $25 \mu \mathrm{M}$ or $50 \mu \mathrm{M}$ plus $10 \mu \mathrm{g} / \mathrm{mL}$ LPS, with or without $10 \mu \mathrm{M}$ Lapaquistat (LAPA). Data are expressed in $\mathrm{pg} / \mathrm{mL}$ and indicated as mean \pm SD of three independent experiments analyzed with ANOVA and corrected with Bonferroni post-test: ${ }^{* *} p<0.01,{ }^{* * *} p<0.001,{ }^{* * * *} p<0.0001$. UNTR: untreated.

\subsection{Lapaquistat Counteracted the Mitochondrial Damage Induced by LPS and Alendronate}

In order to investigate the protective role of Lapaquistat towards the cellular compartments involved in the mevalonate pathway, the morphological changes of RAW 264.7 cells were analyzed using a transmission electron microscope (TEM).

In the control condition (Figure 6a), cells showed a normal morphology, plasmatic and internal membranes were intact, cytoplasmic organelles and chromatin displayed a regular shape. As shown in Figure 6a, the mitochondria were round to oval in shape and presented well-defined cristae. 


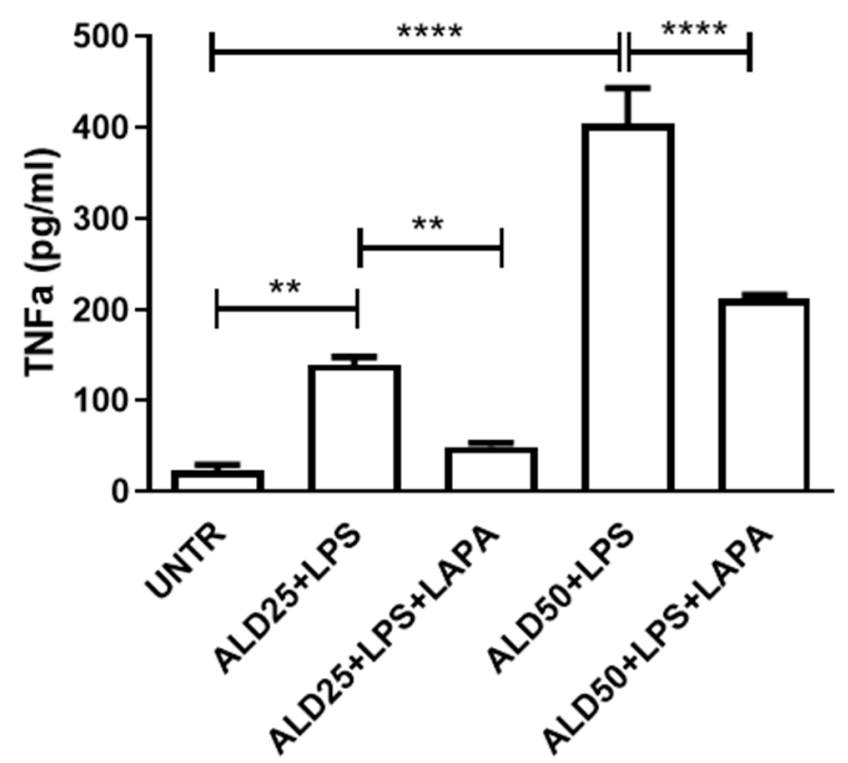

Figure 5. Analysis of TNF- $\alpha$ levels on supernatants of RAW 264.7 treated with ALD $25 \mu \mathrm{M}$ or $50 \mu \mathrm{M}$ plus $10 \mu \mathrm{g} / \mathrm{mL}$ LPS, with or without $10 \mu \mathrm{M}$ Lapaquistat (LAPA). Data are expressed in $\mathrm{pg} / \mathrm{mL}$ and indicated as mean \pm SD of three independent experiments analyzed with ANOVA and corrected with Bonferroni post-test: ${ }^{* *} p<0.01,{ }^{* * * *} p<0.0001$. UNTR: untreated.
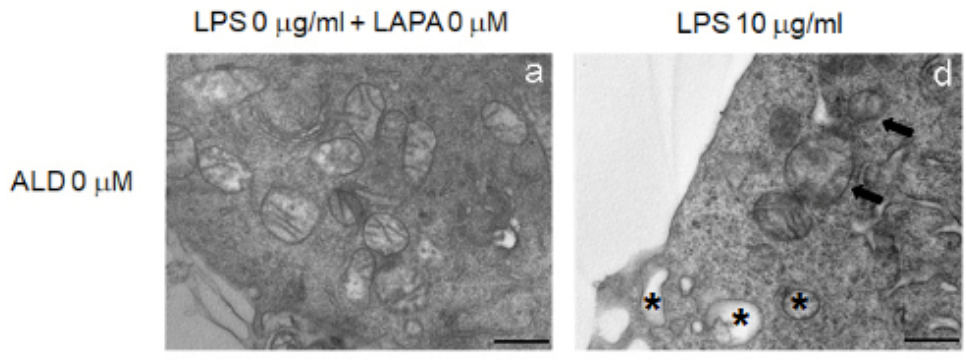

LPS $10 \mu \mathrm{g} / \mathrm{ml}+$ LAPA $10 \mu \mathrm{M}$
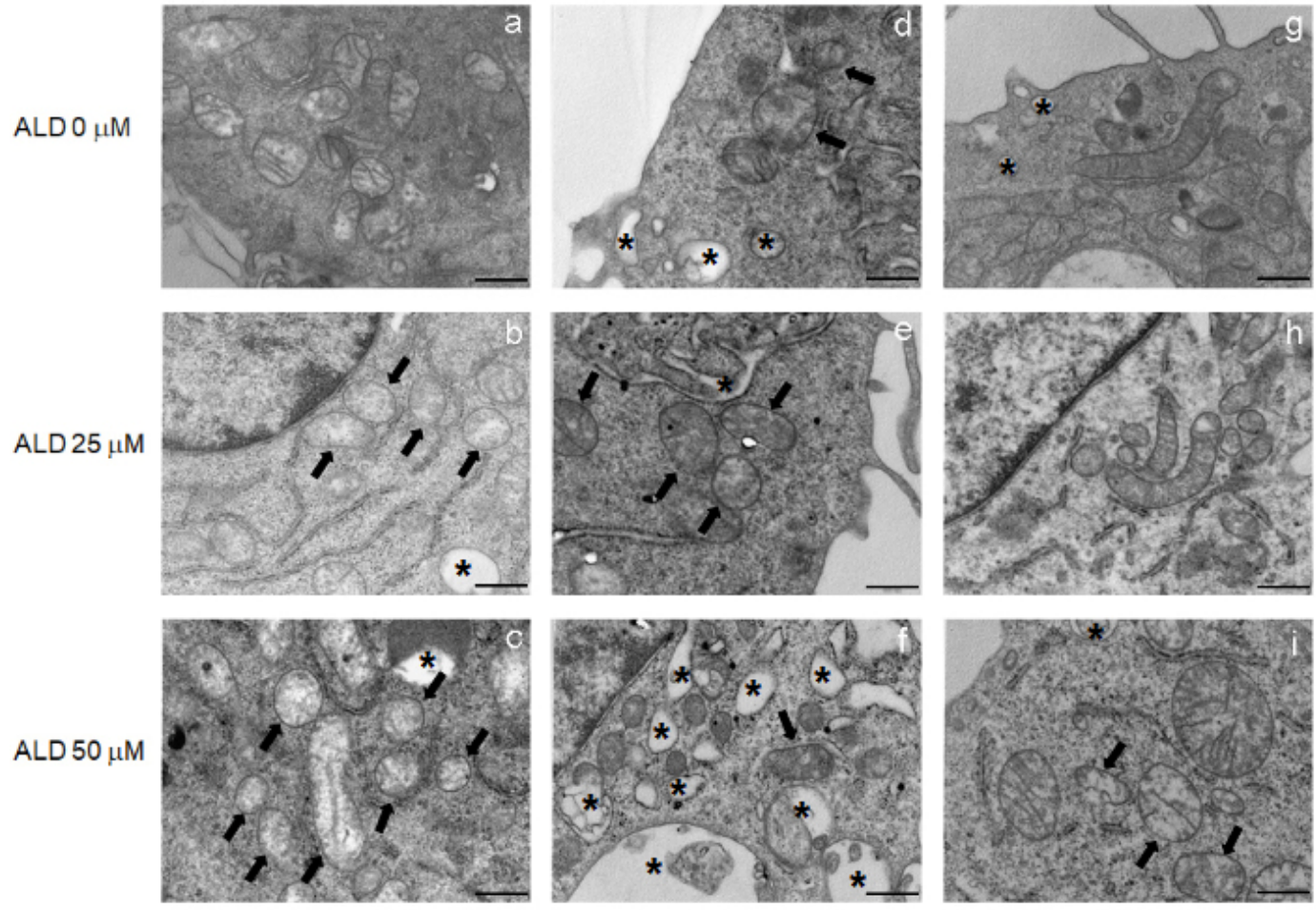

Figure 6. Morphological analysis of RAW 264.7 cells treated with ALD $25 \mu \mathrm{M}$ or $50 \mu \mathrm{M}$ plus $10 \mu \mathrm{g} / \mathrm{mL}$ LPS, with or without $10 \mu \mathrm{M}$ Lapaquistat (LAPA). The ultrastructure of the cells was detected by transmission electron microscope (TEM). (a-i) Representative TEM images of RAW 264.7 cells treated as indicated in the figure labels, 25,000× magnification. Black arrows: swollen mitochondria. Asterisks: cytoplasmic vacuolations. Scale bars: $500 \mathrm{~nm}$.

The addition of ALD $25 \mu \mathrm{M}$ (Figure $6 \mathrm{~b}$ ) and ALD $50 \mu \mathrm{M}$ (Figure $6 \mathrm{c}$ ) for $48 \mathrm{~h}$ induced morphological modification, especially in mitochondria, directly related to the concentration of the bisphosphonate. A fraction of the mitochondria appeared modified with 
damaged cristae, mainly in the ALD $50 \mu \mathrm{M}$ treatment. The treatment with LPS, added the last $24 \mathrm{~h}$ of incubation with ALD $25 \mu \mathrm{M}$ (Figure 6e) and ALD $50 \mu \mathrm{M}$ (Figure 6f), induced an extensive cytoplasmic vacuolation, more evident in ALD $50 \mu \mathrm{M}+\mathrm{LPS}$, and the presence of swollen mitochondria with disrupted cristae. Cells were, then, treated with Lapaquistat alone or in combination with LPS and ALD $25 \mu \mathrm{M}$ or ALD $50 \mu \mathrm{M}$. The morphological analyses showed that Lapaquistat was able to counteract the effects of LPS and ALD (Figure $6 \mathrm{~h}, \mathrm{i}$ ) and that this activity was particularly evident in cells treated with a lower concentration of ALD (Figure 6h). RAW 264.7 treated with ALD $25 \mu \mathrm{M}+$ LPS in combination with Lapaquistat displayed a reduction in the cytoplasmic vacuolation and most mitochondria with a normal morphology and well-defined cristae. The effect of Lapaquistat on ALD $50 \mu \mathrm{M}$ + LPS treated cells was less evident, but cells still showed lower cellular damage and the presence of mitochondria with very clearly visible ridges, in addition to mitochondria that appeared empty with no cristae.

\subsection{Lapaquistat Inhibits Autophagy in RAW 264.7 Cells}

Next, to investigate the mechanism of Lapaquistat, we further examined autophagy using TEM to visualize the presence of autophagosomes and autophagic compartments, based on their distinct ultrastructure [23]. As shown in Figure 7A (panel a), few autophagosomes were present in RAW 264.7 in the control condition. In samples treated with ALD $25 \mu \mathrm{M}$, we observed an increase in the number of cells presenting double membrane vesicles in the cytoplasm, indicative of autophagy (Figure 7A, panel b). The addition of LPS in combination with ALD $25 \mu \mathrm{M}$ resulted in a strong increase in autophagosomes, with the presence of mitochondria surrounded by a double membrane typical of mitophagy [24], and autophagic compartments (Figure 7A, panel c). As shown in Figure 7A (panel d), the contemporary treatment with Lapaquistat reduced the presence of autophagosomes in the cytoplasm, suggesting the blockade of autophagy as a potential mechanism of action.

Starting from these evident morphological observations, we tried to analyze the autophagy-related protein LC3 by a Western blot analysis. As shown in Figure 7B, the treatment with LPS and ALD $25 \mu \mathrm{M}$ induced a great decrease in LC3A/B-I protein expression and this decline was partially restored when Lapaquistat was added in combination with ALD $25 \mu \mathrm{M}$ and LPS.

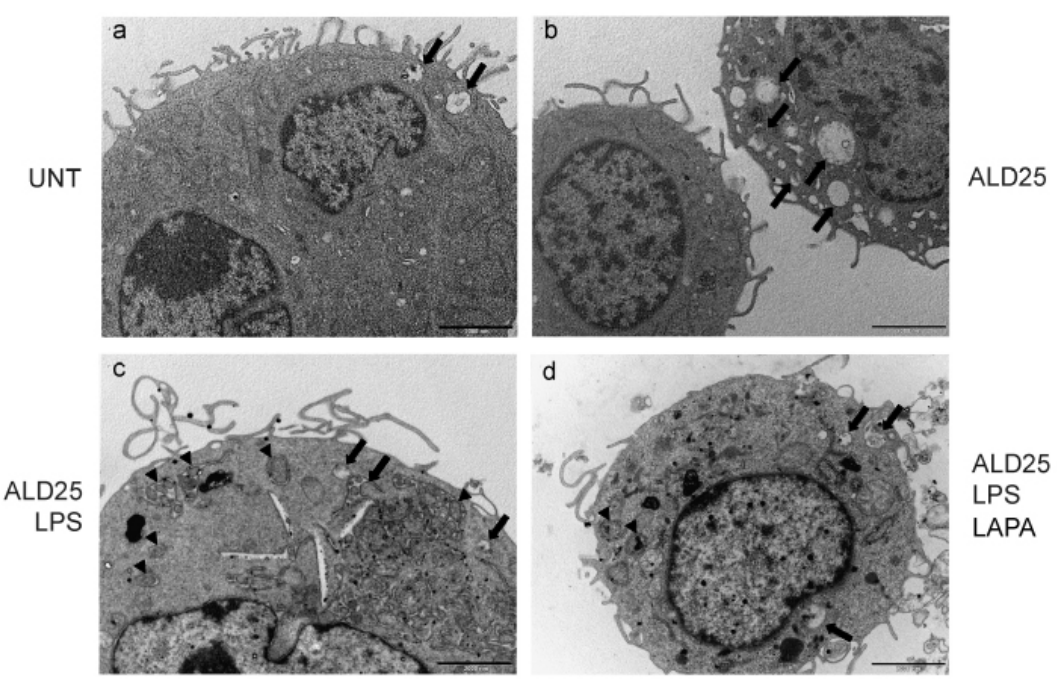

(A)

Figure 7. Cont. 


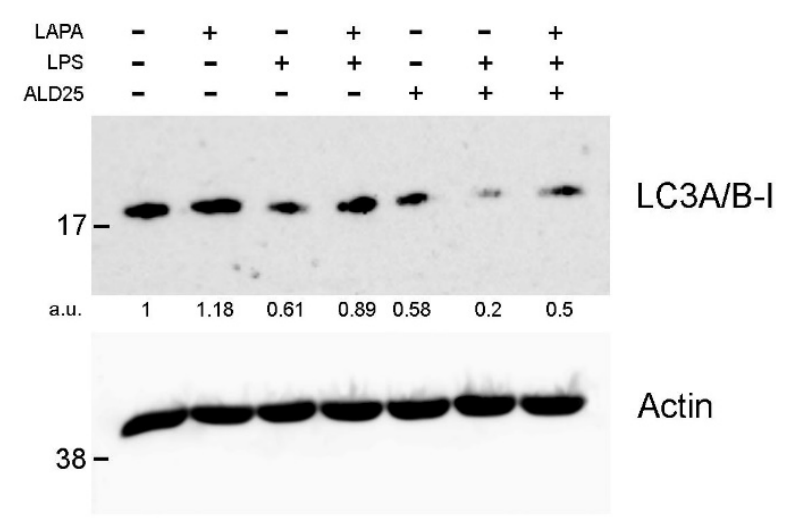

(B)

Figure 7. Evaluation of autophagy in RAW 264.7 treated with ALD $25 \mu \mathrm{M}$ (ALD25) plus $10 \mu \mathrm{g} / \mathrm{mL}$ LPS, with or without $10 \mu \mathrm{M}$ Lapaquistat (LAPA). (A) Representative TEM images of areas of cytoplasm, 8000× magnification. Scale bars: $2000 \mathrm{~nm}$. Black arrowheads: mitophagosomes. Black arrows: autophagic compartments. (B) LC3 protein level was detected by Western blotting analysis. Three independent experiments were conducted; representative blot is shown. $\beta$-actin is shown as loading control. The ratio between LC3A-B I/ $\beta$-actin densitometric quantification is indicated. a.u.: arbitrary unit.

\section{Discussion}

MKD is an autosomal recessive rare disease, associated with mutations in the mevalonate kinase gene, coding for the homonymous enzyme that catalyzes the second step of the metabolic pathway that leads to the biosynthesis of cholesterol. The disease was discovered in 1980 and, to date, more than 250 mutations of this gene have been reported, all resulting in a significant decrease in residual enzyme activity (REA) [25]. The REA variability has allowed, over the years, to define a continuous spectrum of clinical phenotypes ranging from the less severe manifestation of the disease (HIDS) to the more severe one (MA). The pathogenic role of a decrement of mevalonate-derived intermediate compounds in MKD is well recognized: a GGPP-shortage is considered to have a key role in triggering the pathogenesis of the inflammatory MKD phenotype. Since 2016, MKD is no longer considered a treatment-orphan disease. Regulatory agencies have approved the extension of the therapeutic indications of the drug Ilaris (Canakinumab) previously prescribed for CAPS (periodic syndromes associated with cryopyrin), for active systemic juvenile idiopathic arthritis and for other diseases, including MKD, TRAPS (Tumor necrosis factor Receptor-Associated Periodic Syndrome) and FMF (Familial Mediterranean Fever) [11]. Previously, patients with MKD were treated with non-steroidal anti-inflammatory drugs, with poor effectiveness, with glucocorticoids, which were often burdened by serious adverse effects and by dependency, and with anti-cytokine biological drugs (anti TNF- $\alpha$ or anti IL-6) with partial benefit. The response to these pharmacological treatments in patients is variable and difficult to predict. This variability cannot be attributed to distinct MVK genotypes as patients with the same mutation may present significant differences in the clinical phenotype and response to medications. Among the hypotheses for these heterogeneous behaviors, epigenetic factors were suggested to have a discriminating role, but there is no doubt that further insights about MKD pathogenesis are necessary to give an answer to the many questions to establish a consolidated therapeutic line for MKD [26]. The study of TAK- 475 can be inserted precisely in this context. The experimental design used in this study is a consolidated disease model that combines the inhibitory effect of alendronate to block the cholesterol pathway and LPS to mimic the inflammatory stimulus. As shown by the impedancemetry analysis performed in our study, TAK-475 preserved the viability of the experimental cell population treated with alendronate and LPS. In addition, a morphological observation by electron microscopy showed that the changes induced by alendronate could be prevented at least in part by the addition of Lapaquistat. Notably, the 
mitochondrial damage induced by alendronate and LPS treatment, characterized by the presence of swollen mitochondria in the cytoplasm, was almost completely counteracted by Lapaquistat, especially in cells treated with a lower concentration of alendronate. Moreover, a TEM observation evidenced the ability of Lapaquistat to reduce autophagy induced by alendronate and LPS. Autophagy is an active mechanism in the process of cellular stress and in the removal of damaged organelles and intracellular pathogens $[27,28]$. In our model, alendronate and LPS induced an increase in the amount of autophagosomes, frequently containing mitochondria and autophagic compartments, and this induction was counteracted by Lapaquistat. The effect of Lapaquistat on autophagy could be associated with its restoring action on the inflammatory response induced by alendronate and LPS.

Regarding the cytokine profile, as expected, the production of inflammatory cytokines IL-6 and TNF- $\alpha$ was significantly increased following treatment with LPS in cells treated with alendronate in a dose-dependent manner. At both doses of alendronate ( 25 and $50 \mu \mathrm{M})$, TAK- 475 was able to reduce the release of these cytokines, decreasing the inflammatory condition. Transposing these results to MKD, we could hypothesize TAK-475 efficacy limited to the cases with a partial enzyme defect (as in the syndrome with Hyper-IgD), while it seems more unlikely that the drug could be useful for the more serious forms of mevalonic aciduria. Indeed, it is possible that with this drug, as previously reported with other enzymatic modulators, paradoxical effects may be obtained linked to the involvement of different pathogenetic mechanisms in MKD, depending on the severity of the defect.

From all this evidence, the data project us to continue the Lapaquistat studies to evaluate the possibility of its future application in the treatment of MKD and, perhaps, other inflammatory diseases, in view of the repositioning of the drug. It is known that other medications acting on the cholesterol pathway have controversial results when used in patients affected by MKD. For example, statins could lead to a mild improvement in some subjects with MKD [29], but severely worsened the crises in subjects with MA [30]. Alendronate has also been proposed for therapy on the basis of possible benefit in a single patient, but it may also worsen the deficiency in mevalonate-derived isoprenoids, leading to worse inflammation. Indeed, the cholesterol pathway is finely regulated in vivo, and it may be difficult to predict how in vitro studies may reflect on the development of clinical studies. Unfortunately, murine models closely reproducing the human disease are lacking, hindering the translation of new treatments to the clinics. Moreover, considering the fine regulation of the cholesterol pathway, we should also consider the possibility that the blockade of squalene synthase could worsen the lack of post-squalene sterols deficiency in MKD, with possible detrimental clinical consequences. Indeed, while the safety profile of Lapaquistat in healthy subjects is good at low doses, this is not warranted for subjects with MKD, in whom the blockade of squalene synthase may lead to an excessive suppression of the synthesis of sterol derived molecules. The administration of coenzyme Q10, vitamin D and $\mathrm{E}$ is already part of the support therapy in MA-patients and could be even more necessary [31] to reduce the potential adverse effects of the inhibition of squalene synthase, such as myopathy. Finally, the antiviral 25 -hydroxycholesterol $(25-\mathrm{HC})$ has also been found reduced in membranes of subjects with MKD [32] and could be more strongly reduced in the presence of squalene inhibitors.

Thus, more data are still necessary to propose a possible trial of Lapaquistat in subjects with MKD.

\section{Conclusions}

Repositioned drugs, such as Lapaquistat, can have the advantage of reducing development costs and times since pharmacokinetics, toxicology and safety data have been previously collected $[33,34]$. Moreover, although IL-1 inhibitors are showing positive results in clinical trials to control MKD inflammatory phenotypes, TAK-475 may have the theoretical advantage of acting more directly on the biochemical mechanisms involved in the disease over a wider spectrum of clinical manifestations and controlling inflammatory 
disorders. In addition, TAK-475 is an oral available medicine with a short half-life, and this could be a valuable benefit for patients.

TAK-475 acts, as described, downstream of the statins and allows the production of early intermediates of the cholesterol pathway (geranylgeranylation) that would otherwise be limited by the statins. According to this hypothesis, TAK-475 has already been tested in in vivo preclinical studies [20]. To date, no data on the effects of TAK-475 in MKD-patients are available, but some results regarding the effect of TAK- 475 treatment in an in vitro model of MKD are available. Suzuki et al., indeed, recently demonstrated that TAK-475 significantly increases levels of isoprenoids derived from mevalonate (FPP, GGPP and farnesol), in a dose-dependent manner, in human monocytic cells THP-1 [35].

The study of drugs that act on the mevalonate pathway, in general, has allowed us to control the mechanisms that go beyond the production of cholesterol. These results support the hypothesis about a potential pathogenic contiguity between different autoinflammatory diseases; therefore, it is possible that treatment with TAK- 475 may also be useful for other autoinflammatory syndromes.

Supplementary Materials: The following are available online at https:/ /www.mdpi.com/article/10 $.3390 /$ biom11101438/s1, Figure S1: exemplificative figure showing FACS analysis of apoptosis.

Author Contributions: Conceptualization, A.M. and E.R.; methodology, A.M., A.T., E.M. and E.R.; validation, A.M., E.M., E.R. and A.T.; formal analysis, A.M., E.M., E.R. and E.V.; investigation, A.M., E.R., E.M. and E.V.; resources, P.S. and A.T.; writing—original draft preparation, A.M., E.M. and E.R.; writing-review and editing, A.M., E.M., A.T., E.V. and P.S. All authors have read and agreed to the published version of the manuscript.

Funding: This study was supported by a grant from the Institute for Maternal and Child HealthIRCCS "Burlo Garofolo"-Trieste, Italy (RC 24/2017).

Institutional Review Board Statement: Not applicable.

Informed Consent Statement: Not applicable.

Data Availability Statement: Not applicable.

Acknowledgments: This study was supported by a grant from the Institute for Maternal and Child Health-IRCCS "Burlo Garofolo"-Trieste, Italy. We thank Chiara Ghioni and Valentina Boz for their technical support in the cytofluorimetric analysis.

Conflicts of Interest: Alberto Tommasini performed experimental research for Takeda in 2014 about TAK-475, receiving a total amount of 18,000 EUR for research expenses. This previous search activity on TAK-475 had no role in the design of this study; in the collection, analyses, or interpretation of data; in the writing of this manuscript, and in the decision to publish the results. The other authors declare no conflict of interest.

\section{References}

1. Buhaescu, I.; Izzedine, H. Mevalonate pathway: A review of clinical and therapeutical implications. Clin. Biochem. 2007, 40, 575-584. [CrossRef] [PubMed]

2. Drenth, J.P.; Cuisset, L.; Grateau, G.; Vasseur, C.; van de Velde-Visser, S.D.; de Jong, J.G.; Beckmann, J.S.; van der Meer, J.W.; Delpech, M. Mutations in the gene encoding mevalonate kinase cause hyper-IgD and periodic fever syndrome. International Hyper-IgD Study Group. Nat. Genet. 1999, 22, 178-181. [CrossRef] [PubMed]

3. Houten, S.M.; van Woerden, C.S.; Wijburg, F.A.; Wanders, R.J.; Waterham, H.R. Carrier frequency of the V377I (1129G>A) MVK mutation, associated with Hyper-IgD and periodic fever syndrome, in the Netherlands. Eur. J. Hum. Genet. 2003, 11, 196-200. [CrossRef] [PubMed]

4. Hoffmann, G.; Gibson, K.M.; Brandt, I.K.; Bader, P.I.; Wappner, R.S.; Sweetman, L. Mevalonic aciduria-An inborn error of cholesterol and nonsterol isoprene biosynthesis. N. Engl. J. Med. 1986, 314, 1610-1614. [CrossRef] [PubMed]

5. Akula, M.K.; Shi, M.; Jiang, Z.; Foster, C.E.; Miao, D.; Li, A.S.; Zhang, X.; Gavin, R.M.; Forde, S.D.; Germain, G.; et al. Control of the innate immune response by the mevalonate pathway. Nat. Immunol. 2016, 17, 922-929. [CrossRef]

6. Park, Y.H.; Wood, G.; Kastner, D.L.; Chae, J.J. Pyrin inflammasome activation and RhoA signaling in the autoinflammatory diseases FMF and HIDS. Nat. Immunol. 2016, 17, 914-921. [CrossRef]

7. Ibrahim, J.N.; Jéru, I.; Lecron, J.C.; Medlej-Hashim, M. Cytokine signatures in hereditary fever syndromes (HFS). Cytokine Growth Factor Rev. 2017, 33, 19-34. [CrossRef] [PubMed] 
8. Marcuzzi, A.; Zanin, V.; Kleiner, G.; Monasta, L.; Crovella, S. Mouse model of mevalonate kinase deficiency: Comparison of cytokine and chemokine profile with that of human patients. Pediatr. Res. 2013, 74, 266-271. [CrossRef]

9. Marcuzzi, A.; Piscianz, E.; Vecchi Brumatti, L.; Tommasini, A. Mevalonate kinase deficiency: Therapeutic targets, treatments, and outcomes. Expert Opin. Orphan Drugs 2017, 5, 515-524. [CrossRef]

10. Marcuzzi, A.; Loganes, C.; Valencic, E.; Piscianz, E.; Monasta, L.; Bilel, S.; Bortul, R.; Celeghini, C.; Zweyer, M.; Tommasini, A. Neuronal Dysfunction Associated with Cholesterol Deregulation. Int. J. Mol. Sci. 2018, 19, 1523. [CrossRef]

11. De Benedetti, F.; Gattorno, M.; Anton, J.; Ben-Chetrit, E.; Frenkel, J.; Hoffman, H.M.; Koné-Paut, I.; Lachmann, H.J.; Ozen, S.; Simon, A.; et al. Canakinumab for the Treatment of Autoinflammatory Recurrent Fever Syndromes. N. Engl. J. Med. 2018, 378, 1908-1919. [CrossRef]

12. Malcova, H.; Strizova, Z.; Milota, T.; Striz, I.; Sediva, A.; Cebecauerova, D.; Horvath, R. IL-1 Inhibitors in the Treatment of Monogenic Periodic Fever Syndromes: From the Past to the Future Perspectives. Front. Immunol. 2021, 11, 619257. [CrossRef]

13. Schneiders, M.S.; Houten, S.M.; Turkenburg, M.; Wanders, R.J.; Waterham, H.R. Manipulation of isoprenoid biosynthesis as a possible therapeutic option in mevalonate kinase deficiency. Arthritis Rheum. 2006, 54, 2306-2313. [CrossRef] [PubMed]

14. Nishimoto, T.; Amano, Y.; Tozawa, R.; Ishikawa, E.; Imura, Y.; Yukimasa, H.; Sugiyama, Y. Lipid-lowering properties of TAK-475, a squalene synthase inhibitor, in vivo and in vitro. Br. J. Pharmacol. 2003, 139, 911-918. [CrossRef] [PubMed]

15. Amano, Y.; Nishimoto, T.; Tozawa, R.; Ishikawa, E.; Imura, Y.; Sugiyama, Y. Lipid-lowering effects of TAK-475, a squalene synthase inhibitor, in animal models of familial hypercholesterolemia. Eur. J. Pharmacol. 2003, 466, 155-161. [CrossRef]

16. Henneman, L.; van Cruchten, A.G.; Kulik, W.; Waterham, H.R. Inhibition of the isoprenoid biosynthesis pathway; detection of intermediates by UPLC-MS/MS. Biochim. Biophys. Acta 2011, 1811, 227-233. [CrossRef]

17. Shidoji, Y.; Tabata, Y. Unequivocal evidence for endogenous geranylgeranoic acid biosynthesized from mevalonate in mammalian cells. J. Lipid Res. 2019, 60, 579-593. [CrossRef]

18. Marcuzzi, A.; Piscianz, E.; Zweyer, M.; Bortul, R.; Loganes, C.; Girardelli, M.; Baj, G.; Monasta, L.; Celeghini, C. Geranylgeraniol and Neurological Impairment: Involvement of Apoptosis and Mitochondrial Morphology. Int. J. Mol. Sci. 2016, 17, 365. [CrossRef] [PubMed]

19. Marcuzzi, A.; Loganes, C.; Celeghini, C.; Kleiner, G. Repositioning Of Tak-475 in Mevalonate Kinase Disease: Translating Theory into Practice. Curr. Med. Chem. 2018, 25, 2783-2796. [CrossRef]

20. Ebihara, T.; Teshima, K.; Kondo, T.; Tagawa, Y.; Moriwaki, T.; Asahi, S. Pharmacokinetics of TAK-475, a Squalene Synthase Inhibitor, in Rats and Dogs. Drug Res. 2016, 66, 287-292. [CrossRef]

21. Seiki, S.; Frishman, W.H. Pharmacologic inhibition of squalene synthase and other downstream enzymes of the cholesterol synthesis pathway: A new therapeutic approach to treatment of hypercholesterolemia. Cardiol. Rev. 2009, 17, 70-76. [CrossRef]

22. Liao, J.K. Squalene synthase inhibitor lapaquistat acetate: Could anything be better than statins? Circulation 2011, 123, 1925-1928. [CrossRef]

23. Eskelinen, E.L. To be or not to be? Examples of incorrect identification of autophagic compartments in conventional transmission electron microscopy of mammalian cells. Autophagy 2008, 4, 257-260. [CrossRef]

24. Tolkovsky, A.M. Mitophagy. Biochim. Biophys. Acta 2009, 1793, 1508-1515. [CrossRef] [PubMed]

25. Martorana, D.; Bonatti, F.; Mozzoni, P.; Vaglio, A.; Percesepe, A. Monogenic Autoinflammatory Diseases with Mendelian Inheritance: Genes, Mutations, and Genotype/Phenotype Correlations. Front. Immunol. 2017, 8, 344. [CrossRef] [PubMed]

26. Jeyaratnam, J.; Frenkel, J. Management of Mevalonate Kinase Deficiency: A Pediatric Perspective. Front Immunol. 2020, 11, 1150. [CrossRef]

27. Filomeni, G.; De Zio, D.; Cecconi, F. Oxidative stress and autophagy: The clash between damage and metabolic needs. Cell Death Differ. 2015, 22, 377-388. [CrossRef]

28. Bao, M.; Yi, Z.; Fu, Y. Activation of TLR7 Inhibition of Mycobacterium Tuberculosis Survival by Autophagy in RAW 264.7 Macrophages. J. Cell. Biochem. 2017, 118, 4222-4229. [CrossRef] [PubMed]

29. Simon, A.; Drewe, E.; van der Meer, J.W.; Powell, R.J.; Kelley, R.I.; Stalenhoef, A.F.; Drenth, J.P. Simvastatin treatment for inflammatory attacks of the hyperimmunoglobulinemia D and periodic fever syndrome. Clin. Pharmacol. Ther. 2004, 75, 476-483. [CrossRef]

30. Hoffmann, G.F.; Charpentier, C.; Mayatepek, E.; Mancini, J.; Leichsenring, M.; Gibson, K.M.; Divry, P.; Hrebicek, M.; Lehnert, W.; Sartor, K.; et al. Clinical and biochemical phenotype in 11 patients with mevalonic aciduria. Pediatrics 1993, 91, 915-921.

31. Hübner, C.; Hoffmann, G.F.; Charpentier, C.; Gibson, K.M.; Finckh, B.; Puhl, H.; Lehr, H.A.; Kohlschütter, A. Decreased plasma ubiquinone-10 concentration in patients with mevalonate kinase deficiency. Pediatr. Res. 1993, 34, 129-133. [CrossRef] [PubMed]

32. Olsen, B.N.; Schlesinger, P.H.; Ory, D.S.; Baker, N.A. 25-Hydroxycholesterol increases the availability of cholesterol in phospholipid membranes. Biophys. J. 2011, 100, 948-956. [CrossRef] [PubMed]

33. Finsterer, J.; Frank, M. Repurposed drugs in metabolic disorders. Curr. Top. Med. Chem. 2013, 13, 2386-2394. [CrossRef]

34. Lotfi Shahreza, M.; Ghadiri, N.; Mousavi, S.R.; Varshosaz, J.; Green, J.R. A review of network-based approaches to drug repositioning. Brief. Bioinform. 2018, 19, 878-892. [CrossRef]

35. Suzuki, N.; Ito, T.; Matsui, H.; Takizawa, M. Anti-inflammatory and cytoprotective effects of a squalene synthase inhibitor, TAK-475 active metabolite-I, in immune cells simulating mevalonate kinase deficiency (MKD)-like condition. Springerplus 2016, 5, 1429. [CrossRef] [PubMed] 